\title{
Elemental Sulfur as a Versatile Low-Mass- Range Calibration Standard for Laser Desorption Ionization Mass Spectrometry
}

\author{
Andrew Kruegel and Athula B. Attygalle \\ Center for Mass Spectrometry, Department of Chemistry, Chemical Biology and Biomedical Engineering, \\ Stevens Institute of Technology, Hoboken, New Jersey, USA
}

Facile generation of series of singly charged radical anions $\left(\mathrm{S}_{n}^{-\bullet} ; n=1-15\right)$ and cations $\left(\mathrm{S}_{n}^{+\bullet}\right.$; $n=2-11$ ) by direct laser ionization renders elemental sulfur an excellent material for the low-mass-region calibration of time of flight (TOF) mass spectrometers. Upon irradiation by a 337-nm UV laser, elemental sulfur undergoes facile ionization without the need of an additional laser-absorbing matrix. An intense and evenly spaced set of peaks is obtained in both modes. (J Am Soc Mass Spectrom 2010, 21, 112-116) @ 2010 Published by Elsevier Inc. on behalf of American Society for Mass Spectrometry

$\mathrm{O}$ peration of every mass spectrometer entails a procedure called mass axis calibration to convert experimentally measured intensity-time profiles into more practical intensity- $\mathrm{m} / \mathrm{z}$ plots. This modus operandi is accomplished by acquiring a series of peaks for a set of ions of known elemental composition from a single compound or a mixture of compounds. Depending on the ionization technique, varieties of compounds (or their mixtures) have been utilized for calibration. In addition to mass axis calibration, calibrants also provide internal reference peaks for accurate mass determination of ions of unknown composition.

Laser desorption ionization is one of the important ionization methods available for mass spectrometry (LDI-MS). Historically, LDI-MS, and its more extensively used variant, MALDI-MS, are used for mass determination of large molecules [1]. However, many useful applications of LDI-MS in the low mass region $(<m / z 500)$ have also emerged, and the technique has become widely accepted $[2,3]$. For example, organic acids [4, 5], salts of oxyanions [5], amine-based chelating compounds [5], amino acids [6, 7], amino acid derivatives [8], aromatic amines [9], oligosaccharides [10], and pharmaceuticals [11] have been investigated by LDI-MS. However, the calibration of the low mass region is not often straightforward due to the presence of many interfering matrix ions and their fragments. Although a variety of compounds have been used for calibration of LDI mass spectrometers [12-14], good candidates for the low mass region have been particularly sparse.

Address reprint requests to Athula B. Attygalle, Center for Mass Spectrometry, Department of Chemistry, Chemical Biology and Biomedical Engineering, Stevens Institute of Technology, Hoboken, NJ 07030. E-mail: athula. attygalle@stevens.edu
The results reported here outline the use of elemental sulfur as an effective low-mass calibration standard for LDI-MS because it provides a set of evenly spaced peaks for sulfur cluster ions in the low mass range $(\mathrm{m} / \mathrm{z} 0-500)$ observable under both negative and positive modes.

\section{Experimental}

\section{Chemicals}

Sulfur purchased from Matheson Coleman and Bell (Cincinnati, OH, USA) was used without any further purification. The water and toluene used were both HPLC grade and purchased from Pharmco (Brookfield, CT, USA). 2,5-Dihydroxybenzoic acid was purchased from Aldrich Chemical Company (St. Louis, MO, USA).

\section{Sample Preparation}

Sample spots were prepared using sulfur powder suspended in water. A small volume $(1 \mu \mathrm{L})$ of this suspension was spotted on the plate. For the purpose of establishing the consistency of the obtained spectra, several spots were prepared in this manner. Deposits were also prepared by evaporating, at room temperature, a solution $(1 \mu \mathrm{L})$ of sulfur in toluene $(0.5 \mathrm{mg}$ sulfur $/ \mathrm{mL}$ ) without any $\mathrm{pH}$ adjustments. For mass determination of the 2,5dihydroxybenzoate anion, the compound and sulfur were deposited as a solution in toluene.

\section{Mass Spectrometry}

Laser desorption ionization (LDI) mass spectra were recorded using MassLynx software (version 3.5) on a ToFSpec-2E (Micromass, Manchester, UK) equipped 
with a pulsed nitrogen laser (337 nm, pulse width 520 ns, pulse voltage 2,200 V). Source voltage was $20 \mathrm{kV}$ and spectra were recorded in reflectron mode with voltage set at $26 \mathrm{kV}$. Sampling rate was set at $2 \mathrm{GHz}$. The instrument was operated in delayed extraction mode at an extraction voltage of $19,980 \mathrm{~V}$, and focus voltage of $16,000 \mathrm{~V}$. Spectra were acquired from $\mathrm{m} / \mathrm{z} 0$ to 500 without a low mass cut-off setting. The energy of the laser beam was adjusted for the desired ion yield. The fluence of the laser beam was set at different levels at $50 \%$ coarse, and between $40 \%$ and $90 \%$ fine, laser power.

\section{Results and Discussion}

Recently, Hearley et al. showed that laser desorption of elemental sulfur, selenium, tellurium, and phosphorus generate a range of polynuclear ions [15]. For example, sulfur generates a series of ions under both negative and positive ion conditions upon irradiation by a 337 nm UV laser without the assistance of a laser absorbing matrix.

Upon mass analysis, these ions generate spectra with an array of evenly spaced peaks in the low mass region.
For example, Figure 1 and Figure 2 illustrate negative and positive ion laser ionization mass spectra in the range $m / z 0-500$ obtained from deposits of sulfur as an aqueous suspension. Generally, the heterogeneity of sample deposits is considered to give poor spot-to-spot reproducibility, even for the same chemical substance under LDI-TOF conditions. To validate the reproducibility of the peaks under the LDI conditions used in this study, samples were deposited on four different spots on the same target plate, and subjected to laser ablation. The spectra recorded from different sample spots were virtually identical. Moreover, results obtained from spots derived from toluene solutions of sulfur were essentially the same as those from aqueous suspensions.

Raw $m / z$ data obtained directly from the irradiation experiment (Supplementary Figures 1a and 2a, which can be found in the electronic version of this article) were modified by applying a fourth order polynomial curve, based on theoretical values given in Supplementary Table 1 . The $\mathrm{m} / \mathrm{z}$ values obtained after this modification are presented in Supplementary Figures $1 \mathrm{~b}$ and 2b. New spectra acquired using the external mass axis calibration file generated under conditions given in

(a)
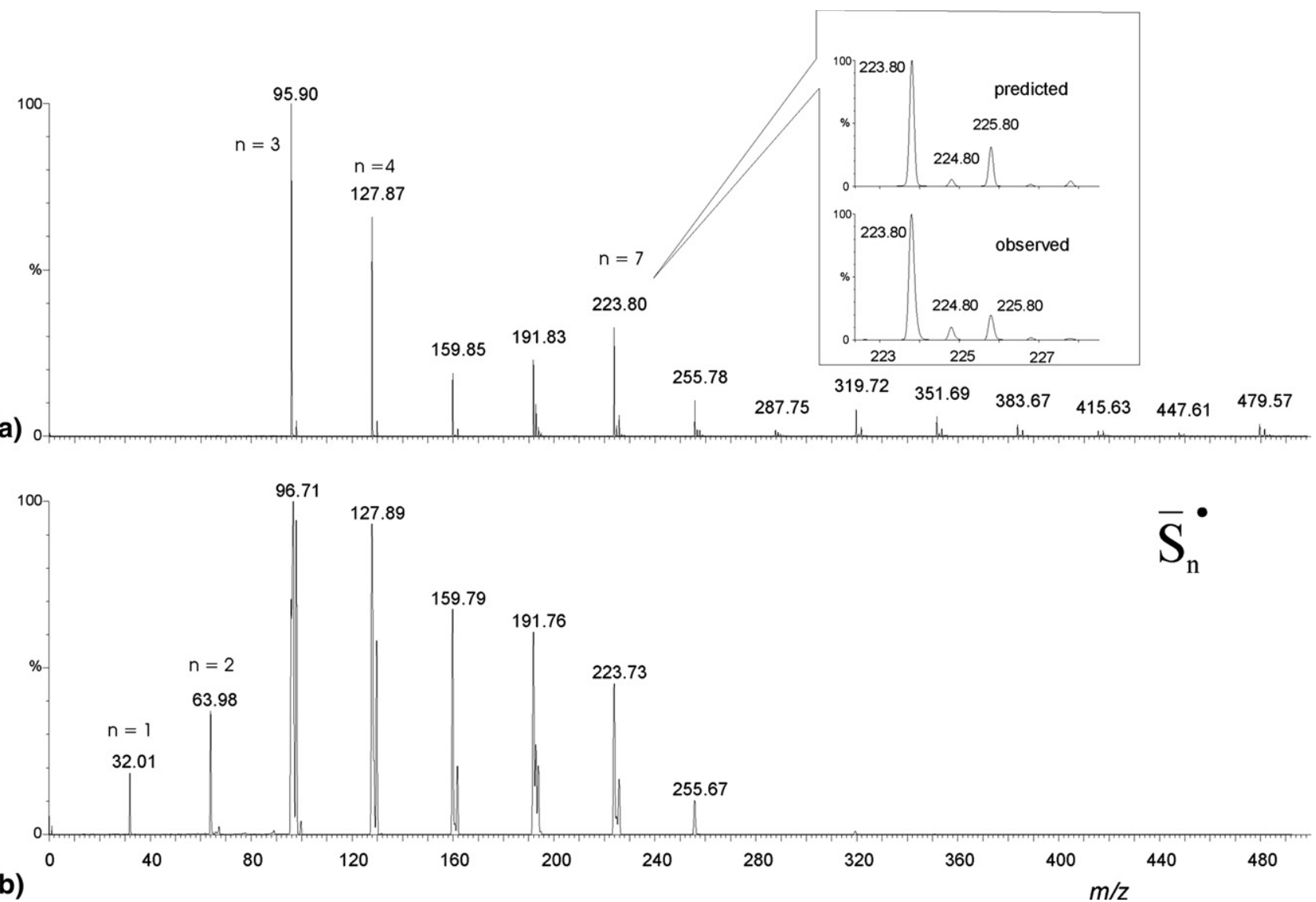

Figure 1. Negative-ion LDI mass spectrometric peak profiles obtained from elemental sulfur under low [(a) laser coarse 50\% and laser fine 45\%], and high [(b) laser coarse 50\% and laser fine 60\%] laser

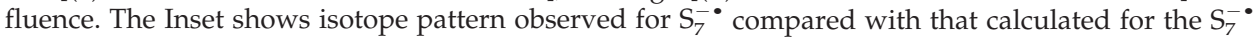
peak cluster. 


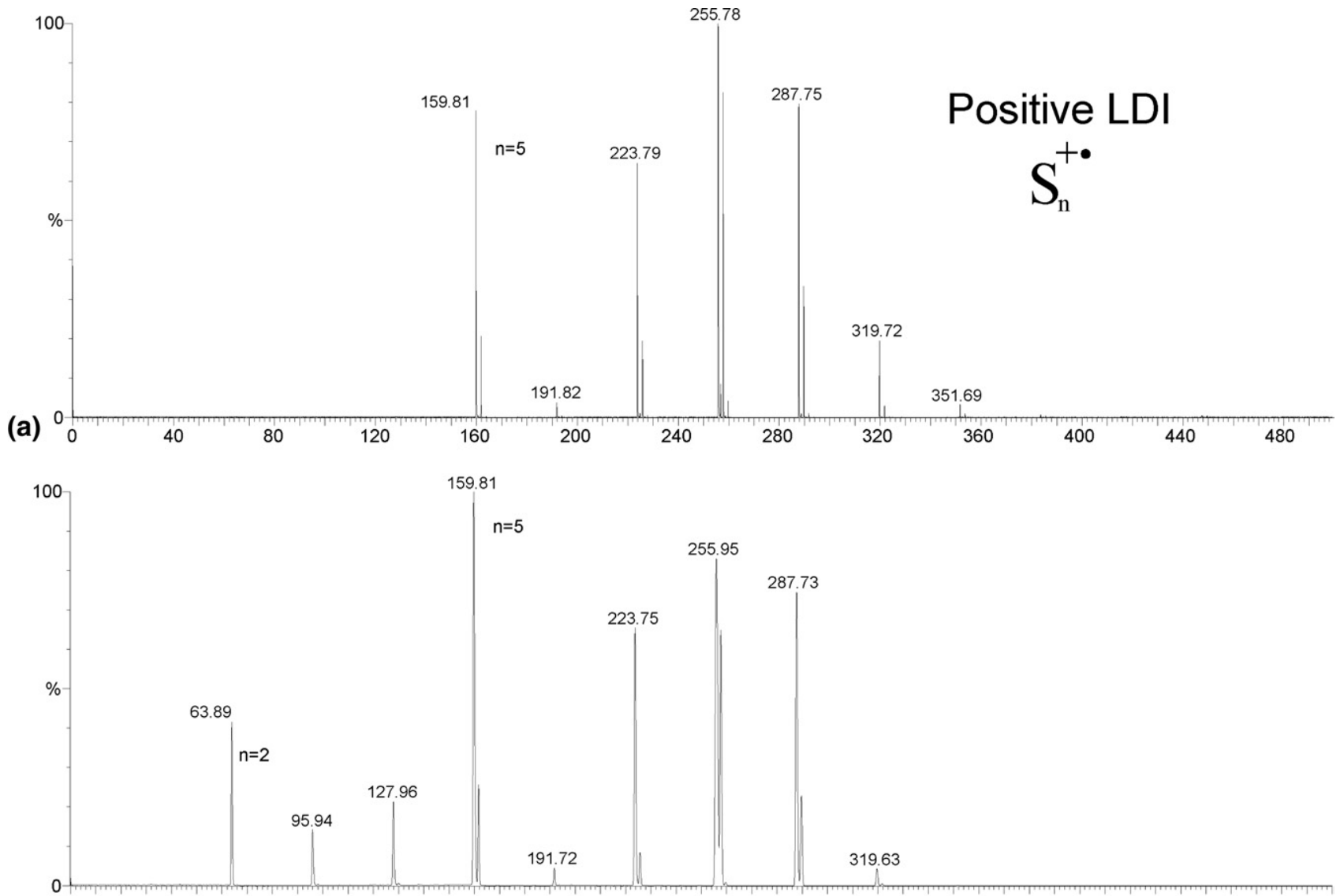

(b)

Figure 2. Positive-ion LDI mass spectrometric peak profiles obtained from elemental sulfur under low [(a) laser coarse $50 \%$ and laser fine $45 \%$ ], and high [(b) laser coarse $50 \%$ and laser fine $75 \%$ ] laser fluence.

Supplementary Figures $1 \mathrm{~b}$ and $2 \mathrm{~b}$ are shown in Supplementary Figures 1c and 2c.

Although sulfur deposits produce series of intense peaks in both ionization modes, the negative ionization is more prolific, as peaks for the entire $\mathrm{S}_{1}^{-\bullet}$ to $\mathrm{S}_{15}^{-\bullet}$ series are observed (Figure 1). In contrast, under positive ion conditions, not as many peaks are observed (Figure 2). To see peaks for the low mass ions, such as $m / z 32$ or 64 , higher laser fluence should be used (laser coarse $50 \%$, and laser fine $60 \%$ ). Unfortunately, high laser fluence leads to deterioration of resolution, shape, and intensity of the peaks for the more abundant ions. This is a well-known limitation of laser ionization-TOF instruments, primarily due to the increased kinetic energy spread of ions in the source before acceleration. Similar dependence on laser power was observed for positive ions as well (Figure 2). However, with optimal adjustments of delayed extraction voltage and time, the resolution of peaks for isotopologues in any cluster could be improved (see inset in Figure 1, which shows acceptable ratios congruent with the theoretical values).

The recognition of sulfur-related peaks in a mass spectrum is possible because this element exists in nature as a mixture of four isotopes $\left[{ }^{32} \mathrm{~S}(94.93 \%),{ }^{33} \mathrm{~S}\right.$
$(0.76 \%),{ }^{34} \mathrm{~S}(4.29 \%)$, and $\left.{ }^{36} \mathrm{~S}(0.02 \%)\right]$. The $2-m / z$ unit separation between the two major peaks in each cluster and their varying relative abundances enable the quick recognition of sulfur peaks, and the determination of the number of sulfur atoms in a given cluster. With fine tuning of acquisition parameters, useful $\left({ }^{32} \mathrm{~S}_{n}\right)^{-}$to $\left({ }^{32} S_{n-1}+{ }^{34} S_{1}\right)^{-\bullet}$ peak ratios can be obtained even for low intensity peaks. For example, the relative abundance ratio of 100:32, observed for a peak cluster under the negative mode of operation, enabled the conclusion that the major peaks in this group represent the isotopologues $\left({ }^{32} \mathrm{~S}_{7}\right)^{-\bullet}$ and $\left({ }^{32} \mathrm{~S}_{6}+{ }^{34} \mathrm{~S}_{1}\right)^{-\bullet}$ (see inset in Figure 1). Most mass spectrometers have computer controlled automatic tuning capabilities. The presence of welldefined isotope peaks in a reference compound is useful for tuning because the instrument can be automated to achieve a target resolution. Because the peak intensity ratios of each cluster are well defined for sulfur, the data system could correctly recognize the identity of peaks even if the raw peaks deviated by several $\mathrm{m} / \mathrm{z}$ units from the expected values (Supplementary Table 1). Although sulfur generates peaks of different intensities, when the high intensity peaks are poorly defined, the operating data system can switch to an isotopologue 


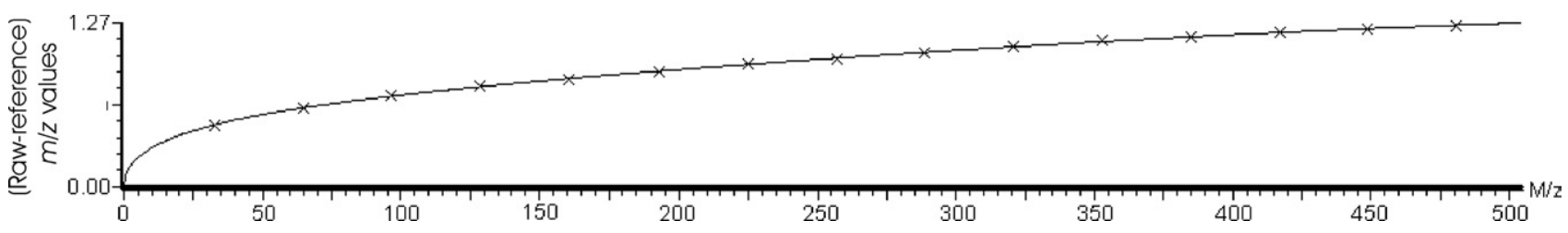

Figure 3. Mass axis calibration curve generated by MassLynx software as a result of a fourth order polynomial fit to the data points generated under negative ion conditions. The ordinate represents the difference between the raw $m / z$ value and the theoretical $m / z$ for each data point (indicated by " $\mathrm{x}^{\prime \prime} \mathrm{s}$ ).

peak of lower intensity to obtain better calibration results. This is particularly important when accurate masses are being measured [16].

An effective calibrant should provide a series of well defined mass markers over the mass range of interest. When many data points are available, a high-order polynomial fit can be used to generate a calibration curve, which can then be used to convert experimentally measured intensity-time profiles into intensity- $\mathrm{m} / \mathrm{z}$ plots. Spectra recorded from sulfur show a range of equally spaced peaks (Figures 1 and 2). Figure 3 shows a fourth-order polynomial fit to a set of data points recoded in negative mode. The ordinate of Figure 3 shows the difference between the raw $m / z$ value and the theoretical $\mathrm{m} / \mathrm{z}$ for each species represented by data points marked as " $x$ "s. The results obtained in this manner showed a maximum deviation of less than 0.01 $\mathrm{m} / \mathrm{z}$ units for each point from the calibration curve. This is a very reasonable level of error for the low-resolution mass spectrometer used in this study.

To verify the applicability of sulfur as an internal mass calibrant for small molecules, a spectrum of 2,5dihydroxybenzoic acid was measured together with that of sulfur. The acquisition was done without the use of an external calibration file. After the acquisition, a mass axis calibration was performed on the sulfur cluster peaks. This calibration process changed the raw $\mathrm{m} / \mathrm{z}$ value for the 2,5-dihydroxybenzoate anion to 153.025 (calculated $\mathrm{m} / \mathrm{z}$ for $\mathrm{C}_{7} \mathrm{H}_{5} \mathrm{O}_{4}$ 153.019). The error of $40 \mathrm{ppm}$ observed in this way was better than the $100 \mathrm{ppm}$ specification expected for the low-resolution ToFSpec-2E mass spectrometer used in this study.

A good calibrant should not leave a memory effect. Spectra recorded from spots other than those specifically coated with sulfur generated no peaks for sulfur clusters. When used as an internal standard, a calibrant must not interfere with or react with the sample. Sulfur did not cause chemical or peak overlapping interferences in our experiments performed with 2,5dihydroxybenzoic acid.

Although the accurate masses and ion intensity ratios presented here are within the expected ranges for the instrument utilized in this investigation, a higher performing mass spectrometer is expected to deliver better results for small molecules when sulfur is used as a mass calibrant.

\section{Conclusions}

Elemental sulfur is an ideal substance for low mass calibration of laser ionization time of flight instruments. Sulfur is not only inexpensive and commercially available in ultra pure form, it is also nontoxic and its sample preparation is extremely simple. A simple dissolution in a solvent, such as toluene, or a suspension in water, is all that is required. The usual sample preparation procedures, such as the addition of laser absorbing matrix chemicals, or buffers, and $\mathrm{pH}$ adjustments with acid or base are unnecessary. MALDI matrices are known to give excessive chemical noise in the low-mass region of spectra. In contrast, sulfur gives no excessive chemical background; therefore, even the very weak peaks can be found and properly assigned. Above all, the same deposit can be used for calibration under both positive and negative polarities, and the peaks do not overlap with the common carbon based ions. When used as an internal mass marker, no significant suppression of the ionization of other analytes was observed. Furthermore, a higher level polynomial fit can be used for calibration because an array of evenly spaced peaks is obtained. In addition, sulfur's lack of memory effects widens its applicability as a low mass calibration standard for LDI-MS. Clearly, sulfur possesses all of the attributes desired from a good calibrant.

\section{Acknowledgments}

The authors acknowledge support for this research by Stevens Institute of Technology.

\section{Appendix A Supplementary Material}

Supplementary material associated with this article may be found in the online version at doi:10.1016/ j.jasms.2009.09.011.

\section{References}

1. Karas, M.; Hillenkamp, F. Laser Desorption Ionization of Proteins with Molecular Masses Exceeding 10,000 Daltons. Anal. Chem. 1988, 60, 2299-2301.

2. Cohen, L. H.; Gusev, A. I. Small Molecule Analysis by MALDI Mass Spectrometry. Anal. Bioanal. Chem. 2002, 373, 571-586.

3. Lidgard, R.; Duncan, M. W. Utility of Matrix-Assisted Laser Desorption/Ionization Time-of-Flight Mass Spectrometry for the Analysis of Low Molecular Weight Compounds. Rapid Commun. Mass Spectrom. $1995,9,128-132$. 
4. Shroff, R.; Muck, A.; Svatoš, A. Analysis of Low Molecular Weight Acids by Negative Mode Matrix-Assisted Laser Desorption/Ionization Time-of-Flight Mass Spectrometry. Rapid Commun. Mass Spectrom. 2007, 21, 3295-3300

5. Goheen, S. C.; Wahl, K. L.; Campbell, J. A.; Hess W. P. Mass Spectrometry of Low Molecular Mass Solids by Matrix-Assisted Laser Desorption/Ionization. J. Mass Spectrom. 1997, 32, 820-828.

6. Hashir, M. A.; Stecher, G.; Mayr, S.; Bonn, G. K. Identification of Amino Acids by Material Enhanced Laser Desorption/Ionization Mass Spectrometry (MELDI-MS) in Positive- and Negative-Ion Mode. Int. J. Mass Spectrom. 2009, 279, 15-24.

7. Gogichaeva, N. V.; Williams, T.; Alterman, M. A. MALDI TOF/TOF Tandem Mass Spectrometry as a New Tool for Amino Acid Analysis. J. Am. Soc. Mass Spectrom. 2007, 18, 279-284.

8. Duncan, M. W.; Matanovic, G.; Cerpa-Poljak, A. Quantitative Analysis of Low Molecular Weight Compounds of Biological Interest by MatrixAssisted Laser Desorption Ionization. Rapid Commun. Mass Spectrom. 1993, 7, 1090-1094.

9. Campbell, J. A.; Wunschel, D. S.; Petersen, C. E. Analysis of Melamine, Cyanuric Acid, Ammelide, and Ammeline Using Matrix-Assisted Laser Desorption Ionization/Time-of-Flight Mass Spectrometry (MALDI/ TOFMS). Anal. Lett. 2007, 40, 3107-3118.

10. Becher, J.; Muck, A.; Mithöfer, A.; Svatoš, A.; Boland, W. Negative Ion Mode Matrix-Assisted Laser Desorption/Ionization Time-of-Flight Mass Spectrometric Analysis of Oligosaccharides Using Halide Adducts and 9-Aminoacridine Matrix. Rapid Commun. Mass Spectrom. 2008, 22, 1153-1158.
11. Shrestha, B.; Li, Y.; Vertes, A. Rapid Analysis of Pharmaceuticals and Excreted Xenobiotic and Endogenous Metabolites with Atmospheric Pressure Infrared MALDI Mass Spectrometry. Metabolomics 2008, 4, 297-311.

12. Harris, W. A.; Janecki, D. J.; Reilly, J. P. Use of Matrix Clusters and Trypsin Autolysis Fragments as Mass Calibrants in Matrix-Assisted Laser Desorption/Ionization Time-of-Flight Mass Spectrometry Rapid Commun. Mass Spectrom. 2002, 16, 1714-1722.

13. Suzuki, H.; Yamagaki, T.; Tachibana, K. Optimization of Matrix and Amount of Ammonium Chloride Additive for Effective Ionization of Neutral Oligosaccharides as Chloride Ion Adducts in Negative-Mode MALDI-TOF Mass Spectrometry. J. Mass Spectrom. Soc. Jpn. 2005, 53, 227-229.

14. Gobom, J.; Mueller, M.; Egelhofer, V.; Theiss, D.; Lehrach, H.; Nordhofft, E. A Calibration Method that Simplifies and Improves Accurate Determination of Peptide Molecular Masses by MALDI-TOF MS. Anal. Chem. 2002, 74, 3915-3923.

15. Hearley, A. K.; Johnson, B. F. G.; McIndoe, J. S.; Tuck, D. G. Mass Spectrometric Identification of Singly-Charged Anionic and Cationic Sulfur, Selenium, Tellurium, and Phosphorus Species Produced by Laser Ablation. Inorg. Chim. Acta 2002, 334, 105-112.

16. Andal, C.; Bocchini, P.; Pozzi, R.; Galletti, G. C. Accurate Mass Measurement of Synthetic Analogues of Prazosin by Matrix-Assisted Laser Desorption/Ionization Time-of-Flight Mass Spectrometry. Rapid Commun. Mass Spectrom. 2001, 15, 665-669. 